\title{
QUALIDADES TECNOLÓGICAS DA CANA-PLANTA E DA CANA-SOCA EM FUNÇÃO DA APLICAÇÃO DA ESCÓRIA DE SIDERURGIA E DO CALCÁRIO ${ }^{1}$
}

\author{
TECHNOLOGICAL QUALITIES OF THE SUGAR-CANE, CANE AND RATOON \\ PLANT, AS A RESULT OF THE APPLICATION OF SLAG AND LIMESTONE
}

\author{
Renato de Mello PRADO ${ }^{2}$
}

\begin{abstract}
RESUMO
Com o objetivo de avaliar o efeito da escória de siderurgia sobre as qualidades tecnológicas da cana-de-açúcar, comparado com o calcário, foi conduzido um experimento de campo durante os dois primeiros cortes da cana-de-açúcar (variedade SP80-1842) efetuados nos anos agrícolas 1998/99 e 1999/00, em Ituverava, região norte do Estado de São Paulo, em Latossolo Vermelho Amarelo álico. Os tratamentos consistiram de dois corretivos, o calcário calcítico e a escória de siderurgia, aplicados em três doses equivalentes a $\mathrm{CaCO}_{3}$, visando elevar a saturação por bases para $50 \% ; 75 \%$ e $100 \%$, além da testemunha sem corretivo. Por ocasião da colheita da cana-planta e da cana-soca aos 12 e 24 meses após o plantio, respectivamente, realizaram-se as análises tecnológicas (brix; pol; pureza e fibra), cálculo do açúcar teórico recuperável (ATR) da cana e da área, e correlação do ATR por área com o rendimento de colmos. Os resultados permitem concluir que nem a escória de siderurgia nem o calcário afetaram significativamente as qualidades tecnológicas avaliadas dos colmos da cana-de-açúcar. No entanto, pelos estudos de regressão, a aplicação exclusiva de escória resultou na cana-de-açúcar em uma relação linear entre as variáveis rendimento de colmos e de açúcar por hectare, atingindo na cana-soca o rendimento de açúcar por hectare $22 \%$ superior ao calcário.

Palavras-chave: resíduo siderúrgico, calagem, rendimento industrial, rendimento de açúcar por hectare, cana-de-açúcar.
\end{abstract}

\begin{abstract}
In the aim of evaluating the effect of slag on the technological qualities of sugar-cane, in comparison to limestone, a field experiment was conducted during the first two cuttings of sugarcane (variety SP80-1842), during the agricultural years of 1998/99 and 1999/00), planted in Ituverava, northern region of São Paulo State, in red-yellow dystrophic latesoil. Treatments consisted of two corrective agents, calcitic limestone and slag, applied in three doses equivalent to $\mathrm{CaCO}_{3}$, aiming an increase of the base saturation to 50,75 and $100 \%$, in addition to the control without corrective agent. On the occasion of harvest of cane and the ratoon plant, of 12 and 24 months after planting, respectively, the techonological analyses (brix; pol; purity and fibre), calculation of the theoretically recoverable sugar (ATR) of the sugar-cane and the area, and correlation of the ATR per area with the culm production, were carried out. The results show that with regard neither the slag nor the limestone affected the technological qualities evaluated of the sugar-cane. However according to the regression studies, application of only slag resulted for the plant cane in a linear relationship between the two variables culm production and sugar yield per hectare. With regard to the ratoon, the sugar yield per hectare was $22 \%$ higher when slag was applied, in comparison to limestone.
\end{abstract}

Key words: metallurgical residue, liming, industrial yield, sugar/hectare yield, sugar cane.

\footnotetext{
1 Parte da Dissertação de Mestrado do primeiro autor, apresentada à Faculdade de Engenharia de llha Solteira/UNESP.

2 Engenheiro Agrônomo, M.Sc., Doutorando, Universidade Paulista Júlio de Mesquita Filho / Faculdade de Ciências Agrárias e Veterinárias de Jaboticabal, Departamento de Solos e Adubos, Via de Acesso Professor Paulo Donato Castellane, s/n, CEP 14870-000, Jaboticabal, SP, Bolsista da FAPESP. E-mail: rmprado@fcav.unesp.br
} 


\section{INTRODUÇÃO}

A cultura da cana-de-açúcar assume grande importância econômica na região Centro-Sul do Brasil em função de possuir a maior área cultivada e a maior produtividade. Isso coloca o Brasil como maior produtor mundial de açúcar e pioneiro na produção de álcool.

Com a expansão da cana-de-açúcar em função dos incentivos econômicos na década de 1980, muitas das áreas marginais de elevada acidez e de baixa fertilidade para cultivo foram incorporadas às áreas das usinas e destilarias. Nessa mesma época houve grande expansão do parque siderúrgico nacional pela maior demanda por ferro e aço dos diversos setores da economia como transporte, construção civil e indústrias automobilísticas, entre outras.

Para a indústria siderúrgica produzir uma tonelada de ferro-gusa e do aço tem como resíduo 0,75 e 0,64 tonelada de escória de siderurgia de alto forno e de aciária respectivamente [4]. Esse fato gerou significativo volume de escória de siderurgia, praticamente sub-utilizada. Tem sido colocado que o uso agronômico desse resíduo, constitui uma forma de destino mais econômico e com menores reflexos para o ambiente. Nesse sentido alguns experimentos conduzidos no Brasil com culturas anuais atestam seu efeito corretivo e como fonte de alguns nutrientes como $\mathrm{Ca}, \mathrm{Mg}, \mathrm{Zn}, \mathrm{B}, \mathrm{Fe}$ e $\mathrm{Mn}$ [13] e, ainda, que a presença do ânion $\mathrm{H}_{3} \mathrm{SiO}_{4}{ }^{-}$exerce efeito competitivo com $0 \mathrm{H}_{2} \mathrm{PO}_{4}^{-}$, reduzindo a adsorção ou aumentando a disponibilidade de fósforo no solo [15]. Assim, esses resíduos siderúrgicos podem ser utilizados em solos ácidos de baixa fertilidade, melhorando suas características químicas e com reflexos na produtividade e na qualidade dos produtos agrícolas, conseqüentemente resolvendo um problema ambiental em torno das indústrias siderúrgicas que acumulam esses resíduos.

Uma das culturas de maior potencial para consumir as escórias de siderurgia é a cana-deaçúcar, em função da sua tradição na reciclagem de resíduos, da estrutura organizada do setor e da vasta área cultivada. No entanto, no Brasil inexistem estudos com a escória de siderurgia visando avaliar a resposta da cultura da cana-de-açúcar quanto ao rendimento industrial. No Estado da Flórida, Estados Unidos, a utilização da escória de siderurgia tem influenciado positivamente as qualidades tecnológicas da cana-de-açúcar, resultando em maior produção de açúcar por área $[3,6]$.

No sentido de contribuir com o assunto foi desenvolvida a presente pesquisa objetivando estudar a influência da aplicação de escória de siderurgia, comparada a do calcário, nas qualidades tecnológicas e relação do rendimento de colmos e o rendimento industrial da cana-planta e da cana-soca.

\section{METODOLOGIA}

O trabalho de campo foi realizado na área experimental da Fazenda Nossa Senhora Aparecida, no município de Ituverava (SP), cujo clima é mesotérmico tipo Cwa, pelo sistema Köppen, em um Latossolo Vermelho-Amarelo álico, classificado, segundo EMBRAPA (1988) [7]. Suas características químicas eram: matéria orgânica $=24 \mathrm{~g} \cdot \mathrm{dm}^{-3} ; \mathrm{pH}$ em $\mathrm{CaCl}_{2}$ 0,01 mol.L-1 $(1: 2,5)=4,6 ;$ P-resina $=5 \mathrm{mg} \cdot \mathrm{dm}^{-}$ 3; $\mathrm{K}=0,4 \mathrm{mmol}_{\mathrm{c}} \cdot \mathrm{dm}^{-3} ; \mathrm{Ca}=8,0 \mathrm{mmol}_{\mathrm{C}} \cdot \mathrm{dm}^{-3} ; \mathrm{Mg}=$ $5,0 \mathrm{mmol}_{\mathrm{c}} \cdot \mathrm{dm}^{-3} ; \mathrm{H}+\mathrm{Al}=34,0 \mathrm{mmol}_{\mathrm{c}} \cdot \mathrm{dm}^{-3} \mathrm{e} \mathrm{V}=26 \%$, avaliadas segundo o método descrito por Raij $\mathrm{e}$ Quaggio (1983) [16].

A escória de siderurgia utilizada foi de aciária básica da siderúrgica Dedini, produtora de aço, do município de Piracicaba, SP, cujas características químicas eram: $\mathrm{Cu}=0,4 ; \mathrm{Zn}=0,9 ; \mathrm{B}=0,3 ; \mathrm{Mn}=$ 24,$7 ; \mathrm{Fe}=261,8 \mathrm{~g} \cdot \mathrm{kg}^{-1}$, analisadas em extrato de DTPA, exceto o $\mathrm{B}$ que foi por água quente; $\mathrm{CaO}=$ 252 g. kg ${ }^{-1} ; \quad \mathrm{MgO}=25 \mathrm{~g} \cdot \mathrm{kg}^{-1} ; \mathrm{PN}$ (Poder de Neutralização $)=51,4 \%$; RE (Reatividade $)=79,4 \%$; e PRNT $=41 \%$. Em um extrato de $\mathrm{HCl} 6 \mathrm{M}$ extraiu-se o $\mathrm{SiO}_{2}$ total $=280 \mathrm{mg} \cdot \mathrm{dm}^{-3}$, que foi determinado por espectrometria de emissão atômica. O calcário utilizado foi 0 calcítico com as seguintes características: $\mathrm{CaO}=372 \mathrm{~g} \cdot \mathrm{kg}^{-1} ; \mathrm{MgO}=27 \mathrm{~g} \cdot \mathrm{kg}^{-1}$; $\mathrm{PN}=87,8 \% ; \mathrm{RE}=73,3 \%$ e o PRNT $=64 \%$. $\mathrm{A}$ escolha desse calcário foi feita porque apresenta teor de óxido de magnésio semelhante ao da escória de siderurgia, inferior a $50 \mathrm{~g} . \mathrm{kg}^{-1}$.

Os tratamentos, em quatro repetições, foram dispostos em blocos casualizados, em fatorial $2 \times 4$, combinando duas fontes de corretivos: calcário calcítico e escória de siderurgia e quatro níveis de correção, compreendendo: a testemunha sem correção, e três doses que objetivaram elevar a saturação por bases para 50,75 e 100\%, correspondendo, respectivamente, em equivalente a $\mathrm{CaCO}_{3}$, a 1,23, 2,52 e 3,8 tha ${ }^{-1}$. Considerando o PRNT de cada corretivo, as doses corresponderam, para o calcário a 1,92, 3,92 e 5,93 tha ${ }^{-1}$ e para a escória de siderurgia a 3,0, 6,12 e 9,27 t.ha- ${ }^{-1}$.

Nos tratamentos com calcário, aplicaram-se os micronutrientes $\mathrm{Cu}, \mathrm{Zn}$ e $\mathrm{B}$ nas doses de 5,91, 8,63 e $3,28 \mathrm{~kg} \mathrm{ha}^{-1}$, respectivamente, para a saturação por bases (V\%) igual a 50 e, assim, proporcionalmente para V\% 75 e 100 . Esse procedimento objetivou equilibrar 0 teor desses micronutrientes nos tratamentos com calcário com os da escória, que os apresenta em sua composição. Como fontes utilizou-se o sulfato de cobre, o sulfato de zinco e o ácido bórico.

Durante o preparo do solo realizaram-se as aplicações da escória de siderurgia e do calcário, manualmente, obedecendo à seguinte seqüência: metade a lanço, em área total, antes da aração com arado de aiveca; e metade a lanço, em área total, depois da aração e antes da gradagem com grade aradora (14x32"). Posteriormente realizou-se o plantio da cana-de-açúcar, variedade SP80-1842 (cana de ano), procurando deixar 15 gemas por metro de sulco, de modo que cada parcela ficou constituída por seis linhas espaçadas de $1,30 \mathrm{~m}$ com $7,5 \mathrm{~m}$ de comprimento, totalizando $58,5 \mathrm{~m}^{2}$ de área. $\mathrm{A}$ área útil da parcela ficou constituída pelas quatro linhas centrais, perfazendo $39,0 \mathrm{~m}^{2}$. 
Como adubação básica, aplicou-se no sulco de plantio, em todos tratamentos, $182 \mathrm{~kg} \cdot \mathrm{ha}^{-1} \mathrm{de}$ $\mathrm{P}_{2} \mathrm{O}_{5}$, na forma de superfosfato simples, $52 \mathrm{~kg} \cdot \mathrm{ha}^{-1}$ de $\mathrm{N}$, na forma de uréia, e $104 \mathrm{~kg} \cdot \mathrm{ha}^{-1}$ de $\mathrm{K}_{2} \mathrm{O}$, na forma de cloreto de potássio. Na mesma ocasião, foram aplicados inseticidas, para controle de pragas, distribuídos em todas as parcelas. Em cobertura utilizou-se $60 \mathrm{~kg} \cdot \mathrm{ha}^{-1}$ de $\mathrm{N}$, na forma de uréia e $60 \mathrm{~kg} \cdot \mathrm{ha}^{-1}$ de $\mathrm{K}_{2} \mathrm{O}$, na forma de cloreto de potássio, aos 42 dias após o plantio. Na cana-soca foram aplicados $100 \mathrm{~kg} \cdot \mathrm{ha}^{-1}$ de $\mathrm{N}$ e $150 \mathrm{~kg} \cdot \mathrm{ha}^{-1}$ de $\mathrm{K}_{2} \mathrm{O}$, aos 30 dias após a emergência dos brotos, incorporados em sulco raso, a $40 \mathrm{~cm}$ da linha e a $5 \mathrm{~cm}$ de profundidade. Para controle de plantas daninhas, adotou-se o químico na cana-planta e o mecânico na cana-soca.

As análises tecnológicas da cana-de-açúcar: pol (sacarose)\%; brix (sólido solúveis)\%; pureza\%; e fibra\% foram realizadas com o apoio da Usina Junqueira, localizada no município de Igarapava-SP. Utilizou-se a metodologia preconizada pelo PLANALSUCAR (1980) [14]. Foram amostrados dez colmos por parcela, aleatoriamente, nas quatro linhas centrais da área útil, na época da colheita (um ano após o plantio e do rebrote da cana-planta e cana-soca, respectivamente).

Com os resultados obtidos nas análises tecnológicas calculou-se o ATR ou açúcar teórico recuperável $\left(\mathrm{kg}^{-1} \mathrm{t}^{-1}\right)$, conforme metodologia da Cooperativa dos Produtores de Açúcar e Álcool do Estado de São Paulo [5] utilizando-se a seguinte equação:

$\operatorname{ATR}\left(\mathrm{kg} \cdot \mathrm{t}^{-1}\right)=(10 \times \mathrm{S}-0,76 \times \mathrm{F}-6,9) \times(5 / 3-200 / 3 \times \mathrm{P})$

onde $\mathrm{ATR}=$ açúcar teórico recuperável em $\mathrm{kg} \cdot \mathrm{t}^{-1}$ de colmos de cana-de-açúcar;

$S($ sacarose $)=$ pol $(\%)$;

$F=$ fibra $(\%) ; e$

$\mathrm{P}=$ pureza $(\%)$.

Os valores de ATR $\left(\mathrm{kg} \cdot \mathrm{t}^{-1}\right)$ foram multiplicados pelos rendimentos de colmos (t.ha ${ }^{-1}$ ) para obtenção do rendimento de açúcar teórico recuperável (t.ha ${ }^{-1}$ ).

Os resultados foram submetidos à análise de variância e, em seguida, foram utilizados modelos estatísticos lineares $(P<0,05)$ [9], especialmente para relação rendimento de colmos e açúcar teórico recuperável para cana-planta e cana-soca.

\section{RESULTADOS E DISCUSSÃO}

\section{Cana-planta}

A Tabela 1 mostra que não houve diferença estatística significativa pelo teste $F(P>0,05)$ para as características tecnológicas (Pol \%, ${ }^{\circ}$ Brix da cana, Pureza \% e Fibra \%) da cana-planta em função dos tratamentos. A falta de resposta da cana-de-açúcar à aplicação de corretivos no solo para tais características tecnológicas foi também observada por Penatti (1987) [12] e Mello e Moura Filho (1998) [11].

Algumas características tecnológicas apresentadas na Tabela 1, como o Pol \% e o ${ }^{\circ}$ Brix da cana-planta, apresentaram valores maiores para o tratamento testemunha, comparado aos dos demais tratamentos, embora sem diferença significativa. Possivelmente, essa tendência exista em razão do efeito diluição, pelo crescimento da planta de cana-de-açúcar.

Como a aplicação de corretivos de maneira geral resulta em aumentos no rendimento de colmos da cana-de-açúcar, pode interferir no rendimento de açúcar por área (ATR área). Assim, correlacionouse por meio da regressão, a produção de colmos e o ATR área, separadamente, para calcário e escória. $\mathrm{Na}$ análise de regressão verificou-se que houve significância para as variáveis envolvidas. A relação do rendimento de colmos da cana-planta com a variável ATR da área apresentou efeito linear apenas para a escória, enquanto que para o calcário não atingiu significância (Figura 1). Pela equação de regressão observa-se, ainda, que a aplicação da escória de siderurgia promoveu aumentos no rendimento, sendo que cada tonelada de colmos de cana-de-açúcar esteve associada à produção de 122 kg de açúcar por hectare.

A relação estreita do rendimento de colmos com a de açúcar, em função da aplicação da escória de siderurgia, foi relatada por diversos autores $[2,3$, 10] que trabalharam com aplicação de escória de siderurgia na cana-de-açúcar em solos de Everglandes, na Flórida, EUA, durante três anos de experimentação. Allorerung (1989) [1] obteve os mesmos resultados em solos ácidos da Indonésia, com aplicação de duas escórias (Java e Japão) nas doses de 1 e 3 t.ha $^{-1}$, resultando em aumento do rendimento de colmos e de açúcar, enquanto que a percentagem de açúcar no colmo não se alterou.

\section{Cana-soca}

Pela Tabela 2 verifica-se que não houve diferença estatística significativa pelo teste $F$ $(P>0,05)$ para as características tecnológicas analisadas, com exceção da percentagem de pureza. Com relação à pureza da cana observou-se que houve diferença apenas para o fator níveis de correção, destacando-se as maiores doses de corretivos.

Observa-se, ainda pela Tabela 2, que o teste F para o açúcar teórico recuperável (ATR) não apresentou efeito significativo para os tratamentos. No entanto, verifica-se que nos tratamentos com aplicação dos corretivos (média do calcário e da escória), o ATR da cana foi menor em relação à testemunha, embora não tenha atingido significância. Essa mesma relação ocorreu na canaplanta (Tabela 1) e também foi descrita por Hurney (1974) [8].

Da mesma forma que na cana-planta, correlacionou-se por meio da regressão, o rendimento de colmos e o ATR área, separadamente 
para o calcário e para a escória. $\mathrm{Na}$ análise de regressão verificou-se que houve significância para as variáveis envolvidas.

A relação do rendimento de colmos com a variável ATR da área apresentou efeito linear tanto para a escória, quanto para o calcário (Figura 2). Portanto, a aplicação dos presentes corretivos resultou em aumento no rendimento de colmos, que, por sua vez, refletiu em acréscimo linear do ATR da área. Pela equação de regressão observa-se que a aplicação da escória de siderurgia e do calcário promoveu aumentos no rendimento de colmos, sendo que para cada tonelada de colmos de canade-açúcar se associa a produção de 103 e $80 \mathrm{~kg}$ de açúcar por hectare, respectivamente. Essa diferença representa $22,3 \%$ de rendimento em açúcar a mais para cada tonelada de colmos produzidos, em função da aplicação da escória de siderurgia comparado a de calcário.

Elawad (1982) [6] estudando o efeito da escória de siderurgia na cana-de-açúcar (canasoca), observou também a relação positiva entre o rendimento da cana-de-açúcar e do açúcar. Isso, segundo o autor, está associado ao incremento no número de colmos por área e na altura dos colmos, em função da aplicação da escória de siderurgia e não, especificamente, nas qualidades tecnológicas (pol e brix) por unidade de colmo.

TABELA 1 - Resultados do teste $F$ e do coeficiente de variação (CV) obtidos da análise de variância e os valores médios das características tecnológicas Brix, Pol, Pureza, Fibra e ATR (Açúcar Teórico Recuperável), na cana-planta, Ituverava, SP, 1998/99

\begin{tabular}{|c|c|c|c|c|c|}
\hline Fator & ${ }^{\circ}$ Brix & Pol (\%) & Pureza (\%) & Fibra (\%) & $\operatorname{ATR}\left(\mathrm{kg} \mathrm{t}^{-1}\right)$ \\
\hline \multicolumn{6}{|l|}{ Corretivo (C) } \\
\hline Calcário & 16,14 & 14,05 & 89,87 & 15,66 & 112,97 \\
\hline Escória & 16,45 & 14,33 & 90,18 & 15,30 & 116,20 \\
\hline $\mathrm{F}$ & $2,34^{\mathrm{ns} 1}$ & $2,06^{\mathrm{ns}}$ & $0,69^{\mathrm{ns}}$ & $2,24^{\mathrm{ns}}$ & $2,19^{\mathrm{ns}}$ \\
\hline \multicolumn{6}{|l|}{ Níveis de correção (NC) } \\
\hline $\mathrm{V} \% 100\left(3,8 \mathrm{tha}^{-1}\right)^{1}$ & 16,26 & 14,20 & 90,24 & 15,47 & 114,83 \\
\hline V\% $75\left(2,52 \mathrm{t} \mathrm{ha}^{-1}\right)$ & 16,05 & 14,05 & 90,18 & 15,63 & 113,28 \\
\hline V\% $50\left(1,23 \mathrm{t} \mathrm{ha}^{-1}\right)$ & 16,43 & 14,22 & 89,74 & 15,48 & 114,56 \\
\hline Testemunha $\left(0 \mathrm{tha}^{-1}\right)$ & 16,44 & 14,31 & 89,88 & 15,34 & 115,67 \\
\hline $\mathrm{F}$ & $0,82^{\mathrm{ns}}$ & $0,32^{\mathrm{ns}}$ & $0,36^{\mathrm{ns}}$ & $0,23^{\mathrm{ns}}$ & $0,21^{\mathrm{ns}}$ \\
\hline \multicolumn{6}{|l|}{$\mathrm{C} \times \mathrm{NC}$} \\
\hline$F$ & $0,79^{\mathrm{ns}}$ & $0,85^{\text {ns }}$ & $0,63^{\mathrm{ns}}$ & $0,37^{\mathrm{ns}}$ & $0,89^{\mathrm{ns}}$ \\
\hline Média & 16,29 & 14,19 & 90,02 & 15,48 & 114,59 \\
\hline CV $(\%)$ & 3,58 & 3,94 & 1,15 & 4,42 & 5,37 \\
\hline
\end{tabular}

TABELA 2 - Resultados do teste $F$ e do coeficiente de variação (CV) obtidos da análise de variância e os valores médios das características tecnológicas Brix, Pol, Pureza, Fibra e ATR (Açúcar Teórico Recuperável) na cana-soca, Ituverava, SP, 1999/2000

\begin{tabular}{|c|c|c|c|c|c|}
\hline Fator & ${ }^{\circ}$ Brix & Pol (\%) & Pureza (\%) & Fibra (\%) & $\operatorname{ATR}\left(\mathrm{kg} \mathrm{t}^{-1}\right)$ \\
\hline \multicolumn{6}{|l|}{ Corretivo (C) } \\
\hline Calcário & 16,51 & 14,26 & 90,12 & 16,34 & 114,38 \\
\hline Escória & $\begin{array}{c}16,72 \\
1,81 \\
\text { ns }\end{array}$ & $\begin{array}{r}14,30 \\
0,06^{n s}\end{array}$ & $\begin{array}{c}90,23 \\
0,44^{n s}\end{array}$ & $\begin{array}{c}16,45 \\
1,47^{\mathrm{ns}}\end{array}$ & $\begin{array}{r}114,88 \\
0,08\end{array}$ \\
\hline \multicolumn{6}{|l|}{ Níveis de correção (NC) } \\
\hline $\mathrm{V} \% 100\left(3,8 \mathrm{tha}^{-1}\right)^{1}$ & 16,54 & 14,14 & 90,91 a & 16,33 & 114,33 \\
\hline V\% $75\left(2,52 \mathrm{t} \mathrm{ha}^{-1}\right)$ & 16,30 & 13,96 & $90,40 \mathrm{ab}$ & 16,49 & 111,90 \\
\hline V\% $50\left(1,23 \mathrm{tha}^{-1}\right)$ & 16,80 & 14,33 & $90,13 \mathrm{~b}$ & 16,51 & 114,93 \\
\hline Testemunha $\left(0 \mathrm{t} \mathrm{ha}^{-1}\right)$ & 16,82 & 14,69 & $89,26 \mathrm{c}$ & 16,24 & 117,37 \\
\hline F & $2,53^{n s}$ & $2,89^{\mathrm{ns}}$ & 16,74 * & $1,88^{\mathrm{ns}}$ & $1,74^{\mathrm{ns}}$ \\
\hline DMS & - & - & 0,67 & - & - \\
\hline \multicolumn{6}{|l|}{$\mathrm{C} \times \mathrm{NC}$} \\
\hline $\mathrm{F}$ & $1,04^{\mathrm{ns}}$ & $0,48^{\mathrm{ns}}$ & $0,51^{\mathrm{ns}}$ & $0,79^{\mathrm{ns}}$ & $0,56^{\mathrm{ns}}$ \\
\hline Média & 16,62 & 14,28 & 90,18 & 16,39 & 114,63 \\
\hline CV (\%) & 2,66 & 3,61 & 0,53 & 1,64 & 4,21 \\
\hline
\end{tabular}




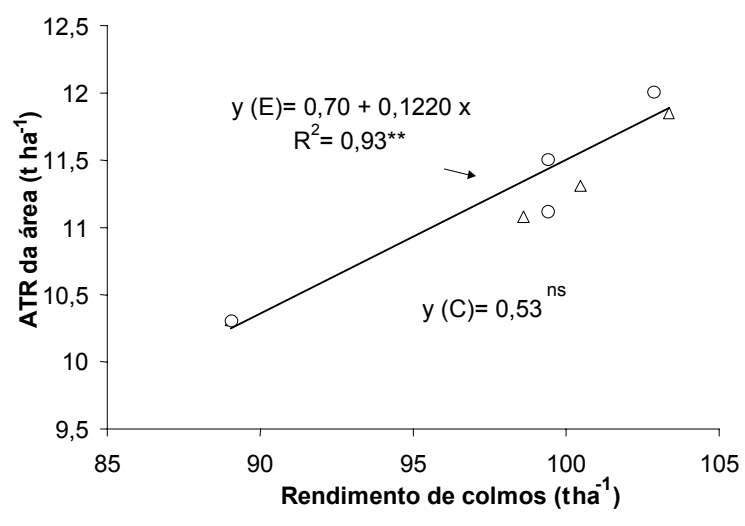

FIGURA 1 - Efeito do rendimento de colmos (t.ha ${ }^{-1}$ ) da cana-planta no rendimento de Açúcar Teórico Recuperável (ATR) (t.ha ${ }^{-1}$ ) com aplicação de calcário (C) e de escória de siderurgia (E), Ituverava,.SP, 1998/99

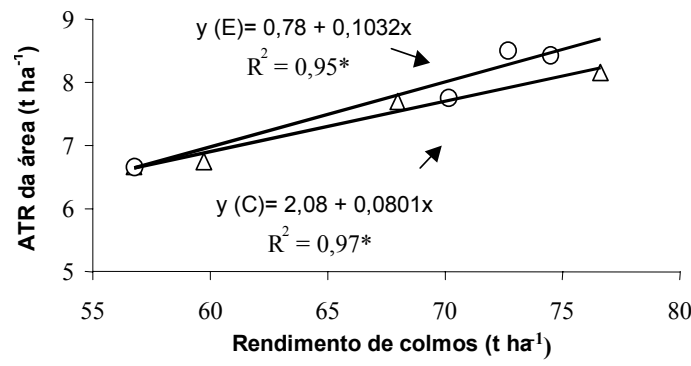

FIGURA 2 - Efeito do rendimento de colmos (t.ha ${ }^{-1}$ ) da cana-soca no rendimento de Açúcar Teórico Recuperável (ATR) (t.ha $\left.{ }^{-1}\right)$, com aplicação de calcário (C) e de escória de siderurgia (E), Ituverava, SP $1999 / 2000$

\section{CONCLUSÕES}

1) As análises tecnológicas (sacarose e sólidos solúveis da cana; pureza, fibra, ATR da cana) dos colmos da cana-planta e cana-soca não foram influenciadas significativamente, exceto a percentagem de pureza na cana-soca, pelo uso da escória de siderurgia e do calcário calcítico.

2) A aplicação exclusiva de escória de siderurgia resultou na cana-de-açúcar em uma relação linear entre as variáveis rendimento de colmos e de açúcar, atingindo na cana-soca o rendimento de açúcar $22 \%$ superior ao calcário.

\section{AGRADECIMENTO}

À FAPESP pela bolsa de mestrado.

\section{REFERÊNCIAS}

[1] ALLORERUNG, D. Influence of steel slag application to red/yellow podzolic soils on soil chemical characteristics, nutrient content and uptake, and yield of sugarcane plantations (Saccharum officinarum L.). Bogor, Indonésia: Faculty of Agriculture / IPB, 1989. p. 14-42. (Bulletin Pusat-Penelitian-Perkebunan-Gula-Indonesia. $N^{\circ}$. 136).

[2] ANDERSON, D. L.; JONES, D. B.; SNYDER, G. H. Response of a rice sugarcane rotation to calcium silicate slag on Everglades Histosols. Agronomy Journal, Madison, v.79, p. 531-535, 1987

[3] ANDERSON, D. L.; SNYDER, G. H.; MARTIN, F. G. Multi-year response of sugarcane to calcium silicate slag on everglades histosols. Agronomy Journal, Madison, v.83, p. 870-874, 1991.

[4] CAMPUS FILHO, M. P. Processos siderúrgicos. In: CAMPUS FILHO, M. P. Introdução à metalurgia extrativa e siderurgia. Campinas: Fundação de Desenvolvimento da Unicamp, 1981. p. 122-153.

[5] COPERSUCAR. Amostragem e análise de cana-deaçúcar. Piracicaba: Centro de Tecnologia COPERSUCAR, 1980. 37p.

[6] ELAWAD, S. H.; GASCHO, G. J.; STREET, J. J. Response of sugarcane to silicate source and rate. I.Growth and yield. Agronomy Journal, Madison, v.74, p. 481-483, 1982.

[7] EMBRAPA. Serviço Nacional de Levantamento e Conservação de Solos I Sistema brasileiro de classificação de solos. Rio de Janeiro: EMBRAPA, 1988. 122 p.

[8] HURNEY, A. P. Effect of silicates on cane growth. Cane Growers Quartely Bulletin, Indooroopilly, v.373, p. 78-80, 1974.

[9] LITTLE, T. M.; HILLS, F. J. Agricultural experimentation: design and analysis. New York: John Wiley, 1978. 350 p.

[10]MARTINS, M. Efeito da calagem na cultura da canade-açúcar (Saccharum spp) em solo sob vegetação de cerrado. Piracicaba, 1991, 85 f. Dissertação (Mestrado em Solos e Nutrição de Plantas) - Escola Superior de Agricultura "Luiz de Queiroz", Universidade de São Paulo.

[11]MELLO, A. J. P.; MOURA FILHO, G. Resposta da cana-de-açúcar ao calcário em Podzólico Amarelo de tabuleiro costeiro sob condições de cultivo mínimo. IN: REUNIÃO BRASILEIRA DE FERTILIDADE DO SOLO E NUTRIÇÃO DE PLANTAS, 23., 1998. Caxambu, Resumos. Lavras: Universidade Federal de Lavras, 1998. p. 596.

[12]PENATTI, C. P.; BONI, P. S.; CONDE, A. J.; SALATA, J. C. Efeito da aplicação de calcário no sulco de plantio de cana-de-açúcar. Piracicaba: Coopersucar, 1987. 35 p. (Boletim Técnico, 39).

[13]PIAU, W. C. Efeitos de escórias de siderurgia em atributos químicos de solos e na cultura do milho (Zea mays L.). Piracicaba, 1995. $124 \mathrm{f}$. Tese (Doutorado em Energia Nuclear na Agricultura) Centro de Energia Nuclear na Agricultura, Universidade de São Paulo.

[14]PLANALSUCAR. Análises tecnológicas da cana-deaçúcar. Araras: Planalsucar, 1980. 34 p.

[15]PRADO, R. M.; FERNANDES, F. M. Efeito do calcário e da escória de siderurgia na disponibilidade de fósforo em Latossolo Vermelho-Escuro e em Areia Quartzosa. Revista de Agricultura, Piracicaba, v.74, n.2, p. 235244, 1999.

[16]RAIJ, B. Van; QUAGGIO, J. A. Métodos de análise de solos para fins de fertilidade. Campinas: Instituto Agronômico de Campinas, 1983. 12 p. (Boletim Técnico, 81).

Recebido para publicação em 10 MAIO 2000 [SA 023/2000] Aceito para publicação em 04 JAN 2002 